\title{
Lyophilisation of influenza, rabies and Marburg lentiviral pseudotype viruses for the development and distribution of a neutralisation -assay-based diagnostic kit
}

\author{
Stuart T. Mather ${ }^{\mathrm{a}}$, Edward Wright ${ }^{\mathrm{b}}$, Simon D. Scott ${ }^{\mathrm{a}}$, Nigel J. Temperton ${ }^{\mathrm{a}, *}$ \\ a Viral Pseudotype Unit (Medway), School of Pharmacy, University of Kent, Chatham Maritime, Kent, UK \\ ${ }^{\mathrm{b}}$ Viral Pseudotype Unit (Fitzrovia), Faculty of Science and Technology, University of Westminster, London, UK
}

Article history:

Received 1 July 2014

Received in revised form

14 September 2014

Accepted 24 September 2014

Available online 5 October 2014

\section{Keywords:}

Retroviral pseudotype viruses

Lyophilisation

Neutralising antibodies

Serological assays

\begin{abstract}
A B S T R A C T
Pseudotype viruses (PVs) are chimeric, replication-deficient virions that mimic wild-type virus entry mechanisms and can be safely employed in neutralisation assays, bypassing the need for high biosafety requirements and performing comparably to established serological assays. However, PV supernatant necessitates $-80^{\circ} \mathrm{C}$ long-term storage and cold-chain maintenance during transport, which limits the scope of dissemination and application throughout resource-limited laboratories. We therefore investigated the effects of lyophilisation on influenza, rabies and Marburg PV stability, with a view to developing a pseudotype virus neutralisation assay (PVNA) based kit suitable for affordable global distribution. Infectivity of each PV was calculated after lyophilisation and immediate reconstitution, as well as subsequent to incubation of freeze-dried pellets at varying temperatures, humidities and timepoints. Integrity of glycoprotein structure following treatment was also assessed by employing lyophilised PVs in downstream PVNAs. In the presence of $0.5 \mathrm{M}$ sucrose-PBS cryoprotectant, each freeze-dried pseudotype was stably stored for 4 weeks at up to $37^{\circ} \mathrm{C}$ and could be neutralised to the same potency as unlyophilised PVs when employed in PVNAs. These results confirm the viability of a freeze-dried PVNA-based kit, which could significantly facilitate low-cost serology for a wide portfolio of emerging infectious viruses.
\end{abstract}

(C) 2014 Elsevier B.V. All rights reserved.

\section{Introduction}

The impact of emerging and re-emerging viral diseases on global health is becoming increasingly apparent year on year. Influenza (family Orthomyxoviridae, genus Influenzavirus A, species Influenza A virus) remains one of the viruses most likely to cause high morbidity and mortality in human populations, after significant outbreaks of H5N1 and H7N9 subtypes beginning in 1997 and 2013 respectively, and the low pathogenic but highly transmissible 2009 H1N1 pandemic virus (Yuen et al., 1998; WHO, 2010; Gao et al., 2013). This threat persists with the first human cases of H6N1 and H10N8, and the recent discovery of diverse H17N10 and H18N11 subtypes in bat reservoirs (Tong et al., 2013; Wei et al., 2013; To et al., 2014). Similarly, rabies (family Rhabdoviridae, genus Lyssavirus, species Rabies virus) is a globally ubiquitous virus, present on all continents

\footnotetext{
* Corresponding author at: Viral Pseudotype Unit, Medway School of Pharmacy, University of Kent, Central Avenue, Chatham Maritime, Kent ME4 4TB, UK. Tel.: +4401634202957.

E-mail address: n.temperton@kent.ac.uk (N.J. Temperton).
}

other than Antarctica, and responsible for over 60,000 deaths per year, primarily of children in resource-limited areas of Asia and Africa (WHO, 2013). Once symptoms occur, rabies has a close to $100 \%$ case fatality rate, the highest of any viral infection. Indeed, only a handful of people have survived following development of clinical symptoms and most of those had neurological sequelae (Jackson, 2013). Sporadic outbreaks of Marburg virus (family Filoviridae, genus Marburgvirus, species Marburg marburgvirus) in the Democratic Republic of the Congo in 1999-2000, and then in Angola in 2004-2005 (respective mortality rates of $83 \%$ and $90 \%$ ), as well as small Ugandan outbreaks more recently, serve to remind us that spillover events into human populations from unexpected viral sources can create serious public health concerns (Brauburger et al., 2012). Therefore, options for monitoring the spread and curtailing the outbreak severity of pathogenic viruses are vitally important.

Serological assays that can detect and quantify antibody responses raised against antigenic surface glycoproteins enable the evaluation of potential vaccines and antiviral treatments, as well as sero-surveillance to monitor the epidemiological movements of a virus, thus contributing to international public health initiatives. Serology compliments direct virus isolation or reverse 
transcription polymerase chain reaction (RT-PCR) diagnosis, by enabling the identification of an acute viral infection after the temporary viremic stage has passed (Papenburg et al., 2011).

However, conventional serological assays possess drawbacks which detrimentally affect their efficiency. Importantly, most require the use of infectious wild-type virus, necessitating expensive, specialised biosafety level 3 or 4 (BSL-3 or -4 ) laboratories which are not readily available, especially in resource-limited areas. Haemagglutination inhibition (HI) assays, used routinely for influenza, suffer from variability caused by different erythrocytes and inhibitory factors, as well as low sensitivity. ELISA-based assays do not require the use of wild-type virus, but are also hindered by low sensitivity and cross-reactivity between samples. Furthermore, both HI and ELISA cannot differentiate between virus neutralising and non-neutralising antibody responses (Mather et al., 2013). Virus neutralisation assays, such as plaque reduction neutralisation test (PRNT) and fluorescent antibody virus neutralisation (FAVN) assay, can measure virus neutralising antibody (VNAb) responses with high sensitivity and specificity levels but also require high biosafety for assay preparation, and in some cases are timeconsuming and suffer from low-throughput (Cliquet et al., 1998; Mather et al., 2013).

A potential solution to these issues is the utilisation of retroviral pseudotype viruses (PVs). PVs are composed of the structural and enzymatic core of one virus combined with heterologous envelope glycoproteins (Temperton and Wright, 2009). Manipulations to the genomic RNA of the lentiviral core create a replication-defective PV that encapsulates a quantifiable reporter gene. Transduction of a permissible target cell line is dependent upon the ability of the envelope glycoprotein to engage its cellular receptor in a process that mimics wild-type virus entry mechanisms. If this is successful, the reporter gene can be integrated into the host cell genome and subsequently expressed. Resultant levels of reporter protein in transduced cells can be measured, giving a readout equivalent to viral titre. Pseudotype virus neutralisation assays (PVNAs) attain comparable, if not higher, sensitivity and specificity results than many traditional serological assays (Desvaux et al., 2012).

In order to maximise the utility of the pseudotype assay system, multiplexing of PVNAs has been demonstrated which permits simultaneous quantification of VNAb responses against several PVs (each harbouring a different reporter gene i.e. renilla and firefly luciferase, or GFP and RFP) in the same assay, sparing valuable reagents such as serum samples (Wright et al., 2010). The flexibility of reporter genes that can be incorporated into PVs further customises the assay. Luciferase and GFP reporters enable highly quantitative readouts but require expensive reagents and/or equipment. However, infection by PVs that encapsulate lacZ (expressing $\beta$-galactosidase) or secreted alkaline phosphatase (SEAP) reporter genes can be quantified by adding colorimetric substrates such as ONPG, CPRG or p-nitrophenyl phosphate and measuring colour change with an ELISA plate reader or by eye (Wright et al., 2009; Kaku et al., 2012).

Multiplexing, as well as selecting 'low-cost' reporter genes, considerably reduces the cost-per-assay burden of the pseudotype platform. However, the high expenses associated in optimal transportation and storage can be an inhibitory obstacle in the international distribution of PVNAs. Despite pseudotype studies being conducted on field serum from resource-poor tropical countries, and reports of viruses that circulate in tropical regions being successfully pseudotyped (Wright et al., 2009; Kishishita et al., 2013), there appear to have been no published studies involving the carrying out of pseudotype neutralisation assays in tropical countries, especially in rudimentary laboratories without air-conditioning or access to reliable freezer units.

The aim of this study was to ascertain the viability of lyophilising pseudotype viruses with a view to developing a PVNA-based kit. Pseudotype stability was monitored after subjection to environmental conditions likely experienced in the production, transit and usage of such a kit, especially to tropical countries. PV titres were also assessed subsequent to lyophilisation and immediate reconstitution, as well as incubating freeze-dried pellets at a variety of temperatures and humidities before reconstitution.

\section{Materials and methods}

\subsection{Viruses and cells}

The virus isolates pseudotyped in this study were influenza A/H5N1/Vietnam/1194/2004 strain (Genbank accession number ABP51976), rabies virus (RABV) strain Evelyn Rokitniki Abseleth (ERA; UniProtKB/Swiss-Prot code ABN11294) and the Lake Victoria strain of Marburg virus (MARV; Genbank accession number DQ447649). Previously, the influenza HA gene and RABV G gene of these isolates were both sub-cloned into the pI.18 expression vector (Cox et al., 2002). The Marburg GP gene within the pCAGGS expression vector was a kind gift from Graham Simmons (Blood Systems Research Institute, San Francisco, CA, USA).

Human embryonic kidney 293T clone 17 cells (HEK293T/17; ATCC CRL-11268) (Pear et al., 1993) were used for all transfections and as a target cell line for titration and neutralisation assays involving H5 pseudotype virus. Baby hamster kidney 21 cells (BHK-21; ATCC CRL-10) (Stoker and MacPherson, 1964) were used as a target cell line for RABV and MARV pseudotype virus assays. Both cell lines were cultured at $5 \% \mathrm{CO}_{2}$ in Dulbecco's Modified Eagle Medium (DMEM) + GlutaMAX (Life Technologies, UK) supplemented with $15 \%$ foetal bovine serum (FBS) and 1\% penicillin/streptomycin (Sigma-Aldrich, UK).

\subsection{Serum samples}

For use in H5 PVNAs, a sample from a panel of ten sera extracted from chickens vaccinated with an inactivated, monovalent, adjuvanted H5N2 vaccine (A/chicken/Mexico/232/94/CPA strain) was selected. Previous studies have confirmed its seropositivity by $\mathrm{HI}$ (a titre of 1:1024 with a homologous H5N2 test antigen) and PVNA, against an H5 A/Vietnam/1194/2004 luciferase PV (Terregino et al., 2010; Molesti et al., 2013). To neutralise RABV pseudotypes, serum was used from a human subject vaccinated on days 0,7 and 21 with the inactivated Rabipur vaccine (Novartis Vaccines, Germany).

\subsection{Production of pseudotype viruses}

The generation of all lentiviral pseudotype viruses was performed as detailed previously (Temperton et al., 2007; Wright et al., 2008). $24 \mathrm{~h}$ prior to transfection, approximately $4 \times 10^{6}$ HEK293T/17 cells were seeded into sterile $10 \mathrm{~cm}^{3}$ tissue culture plates (Nunc ${ }^{\mathrm{TM}}$ Thermo Scientific, UK). The HIV gag-pol plasmid, pCMV- $\Delta 8.91$ (Zufferey et al., 1997) and the firefly luciferase reporter construct pCSFLW (Capecchi et al. (2008) based on pHR'SIN-cPPT-SGW outlined in Demaison et al. (2002)) were transfected simultaneously with either the influenza HA, rabies G or Marburg GP expression vectors at a ratio of 1:1.5:1 (core:reporter:envelope) using the Fugene6 lipid-based reagent (Promega, UK). At $24 \mathrm{~h}$ post-transfection, the cells were incubated with fresh media. For H5 transfections, exogenous recombinant neuraminidase from Clostridium perfringens (Sigma-Aldrich, UK) was also added at this stage. Pseudotype supernatants were harvested at $48 \mathrm{~h}$ after transfection and passed through a $0.45 \mu \mathrm{m}$ pore filter (Millex ${ }^{\circledR}$, Millipore, Billerica, MA, USA), before being prepared for lyophilisation. Remaining supernatant was aliquoted and stored at $-80^{\circ} \mathrm{C}$. 


\subsection{Lyophilisation of pseudotype viruses}

Individual samples of pseudotype virus were mixed with a sucrose-PBS cryoprotectant solution at a $1: 1 \mathrm{v} / \mathrm{v}$ ratio to a $1-0.1 \mathrm{M}$ range of molarities. Importantly, all lyophilisation was carried out in low surface-tension polypropylene microcentrifuge tubes (Caesa Lab, Canada), to prevent binding of the virus glycoproteins to the inside surface of the tubes, and subsequent loss of pseudotype titre, during freeze-drying. Once prepared, virus samples were prefrozen at $-80^{\circ} \mathrm{C}$. Immediately prior to lyophilisation, a second, pierced lid, made of standard polypropylene, was applied to each sample tube to allow for moisture release. All lyophilisation was carried out overnight in a FreeZone 2.5 litre freeze-drying chamber (Labconco, Kansas City, MO, USA) at a temperature of $-50^{\circ} \mathrm{C}$ and a pressure of $<0.133$ mbar. If the lyophilised pellets were stored for a sustained length of time after freeze-drying, the standard polypropylene pierced lid was removed from the sample tube, and the original low surface-tension polypropylene lid was replaced. Likewise, in the instances where the pellets were stored at a constant humidity as well as temperature, the sample tubes were kept in a sealed, humidified incubator unit, controlled by a humidistat. DMEM + GlutaMAX (with the same supplementation as for the cell culture) were attempted for all reconstitution of lyophilised pellets, except for Fig. 6 where distilled, nuclease-free $\mathrm{H}_{2} \mathrm{O}$ was also used.

\subsection{Pseudotype titration and neutralisation assays}

Titration and neutralisation assays were performed in 96-well plates and based upon previously described protocols (Temperton et al., 2007; Wright et al., 2009; Scott et al., 2012), but adapted for the use of reconstituted, lyophilised pseudotype. For titration assays, 1:2 serial dilutions of reconstituted pseudotype were incubated with $1 \times 10^{4}$ HEK293T/17 or BHK-21 cells for 48 hours before measuring relative luminescence units per $\mathrm{ml}(\mathrm{RLU} / \mathrm{ml})$. For the neutralisation assay, serum samples were serially diluted (ranging from $1: 40$ to $1: 81,920$ ) and incubated with $1 \times 10^{6}$ RLU of reconstituted pseudotype (as calculated from the titration assay) for $1 \mathrm{~h}$ at $37^{\circ} \mathrm{C}$ to permit antibody attachment to surface virus glycoproteins. $1 \times 10^{4}$ HEK293T/17 or BHK-21 cells were then added to each well and incubated for 48 hours, prior to taking a chemiluminescent readout. In all instances, Bright-Glo luciferase assay reagent (Promega, UK) and a Glomax 96 luminometer (Promega, UK) were used to quantify luciferase reporter expression.

\subsection{Statistical analysis}

Pseudotype transduction titres were calculated by converting RLU readout values at a range of assay dilutions into $\mathrm{RLU} / \mathrm{ml}$, before determining the arithmetic mean and standard deviation (SD). PVNA raw data was normalised as \% neutralisation between mean values for a virus only control (equivalent to $0 \%$ neutralisation or $100 \%$ infection) and a cell only control (equivalent to $100 \%$ neutralisation or $0 \%$ infection), then $\mathrm{IC}_{50}$ and $\mathrm{IC}_{90}$ values were calculated using non-linear regression analysis (log [inhibitor] vs normalised response - variable slope). All data manipulation was performed on GraphPad Prism 5 (GraphPad software, San Diego, CA, USA).

\section{Results}

High titre lentiviral pseudotype particles were generated bearing the envelope glycoproteins from influenza A/H5N1/Vietnam/1194/2004, rabies ERA and Marburg Lake Victoria strains. Transduction efficiency of the pseudotypes into HEK293T/17 cells (for influenza H5) and BHK-21 cells (for RABV and MARV) was evaluated, and luciferase expression was observed at $2.04 \times 10^{10}, 8.21 \times 10^{9}$ and $7.46 \times 10^{9} \mathrm{RLU} / \mathrm{ml}$, respectively

\section{H5 AVietnam/1194/2004, RABV ERA and MARV Lake Victoria PV titres}

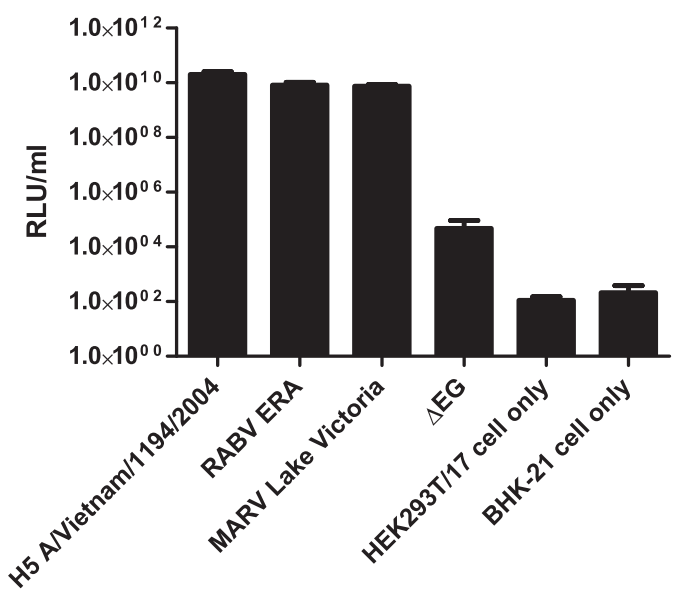

Fig. 1. Infectivity of pseudotyped lentiviral vectors displaying influenza H5 A/Vietnam/1194/2004 HA, RABV ERA G and MARV Lake Victoria GP glycoproteins. Pseudotype transduction titres are expressed as mean \pm SD of relative luminescent units per $\mathrm{ml}(\mathrm{RLU} / \mathrm{ml})$. $\Delta$ envelope glycoprotein, HEK293T/17 cell only and BHK-21 cell only negative controls are also shown.

(Fig. 1). All titration assays included two negative controls: $\Delta$ envelope glycoprotein $(\Delta \mathrm{EG})$, which is a PV bearing no viral envelope glycoprotein, and a non-transduced cell only control.

Next, pseudotype supernatants were mixed with a stepwise dilution series of sucrose-PBS solutions ( $1 \mathrm{M}, 0.5 \mathrm{M}, 0.25 \mathrm{M}, 0.1 \mathrm{M}$ ) which acts as a cryoprotectant during lyophilisation. Supernatant was also lyophilised in pure PBS solution containing no sucrose, which is referred to as $0 \mathrm{M}$ sucrose-PBS. After overnight freezedrying, lyophilised pellets were immediately reconstituted and transduction efficiency measured in a titration assay. Less than $1 \log _{10}$ of decrease in viral titre, measured in RLU/ml, was observed with H5, RABV and MARV pseudotypes at all cryoprotectant concentrations, when compared to their non-lyophilised counterparts (Fig. 2a-c). Levels of titre retention are therefore sufficient for these lyophilised PVs to be taken forward into PVNA assays. As PV titre was retained following reconstitution of recently lyophilised pellets, regardless of sucrose-PBS concentration, freeze-drying for subsequent experiments was carried out at $1 \mathrm{M}, 0.5 \mathrm{M}$ and $0 \mathrm{M}$ cryoprotectant molarities.

To ascertain the stability of PV aliquots stored in the freezedried state, individual lyophilised pellets in $1 \mathrm{M}, 0.5 \mathrm{M}$ and $0 \mathrm{M}$ cryoprotectant were incubated for varying durations at the following temperatures: $-80^{\circ} \mathrm{C},-20^{\circ} \mathrm{C},+4{ }^{\circ} \mathrm{C},+20^{\circ} \mathrm{C},+37^{\circ} \mathrm{C} / 70 \%$ relative humidity $(\mathrm{RH})$ and $+37^{\circ} \mathrm{C} / 95 \% \mathrm{RH}$. After 1,2 and 4 weeks, freeze-dried pellets of PV were reconstituted and titrated as previously described (Sections 2.4 and 2.5) to calculate viral titre in $\mathrm{RLU} / \mathrm{ml}$. Generally, PV titre retention was high for all lyophilised H5 (Fig. 3a-c), RABV (Fig. 4a-c) and MARV (Fig. 5a-c) samples that were stored at the lowest temperatures, but as the storage temperature increased, PV samples freeze-dried in the absence of cryoprotectant degraded significantly, with transduction efficiency decreasing to that of $\triangle \mathrm{EG}$. Interestingly, relative humidity $(\mathrm{RH})$ seems to play a role in viability of lyophilised PV pellets, with $1 \mathrm{M}$ - and $0.5 \mathrm{M}$-cryoprotected samples stored for 4 weeks generally retaining functional virus titre up to $37^{\circ} \mathrm{C}$ and $70 \% \mathrm{RH}$, but heavily degrading in a $95 \%$ humidified atmosphere at the same temperature.

It is possible that reconstituting in supplemented DMEM results in an accumulation of soluble culture medium components in the pseudotype sample which may affect downstream employment 
(A)

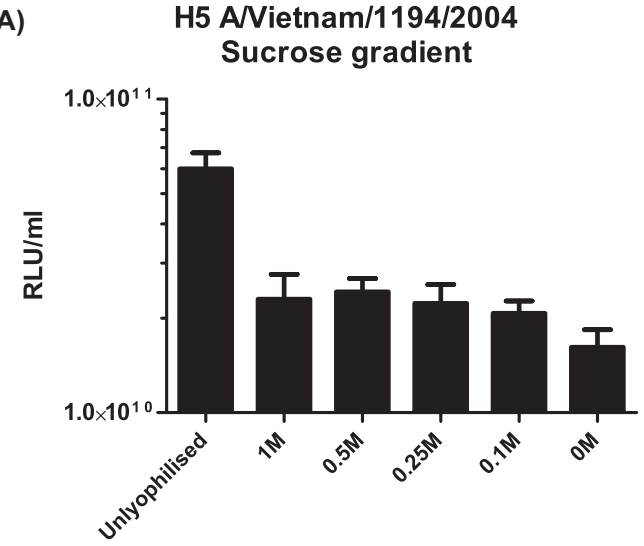

[Sucrose-PBS] (M)
(B)

RABV ERA

Sucrose gradient

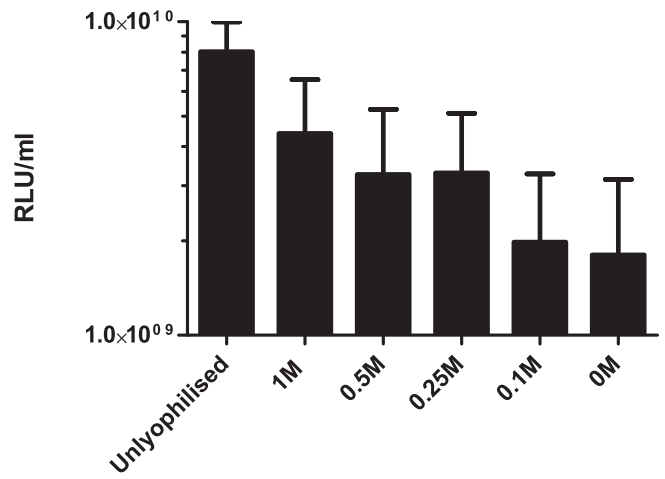

[Sucrose-PBS] (M)

\section{(C) \\ MARV Lake Victoria \\ Sucrose gradient}

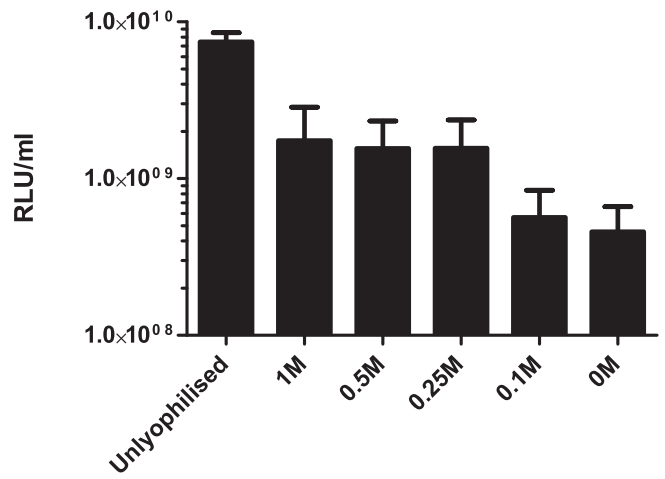

[Sucrose-PBS] (M)

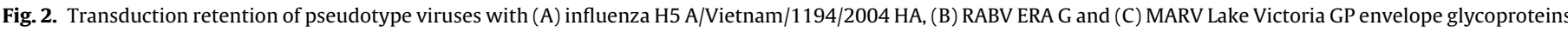

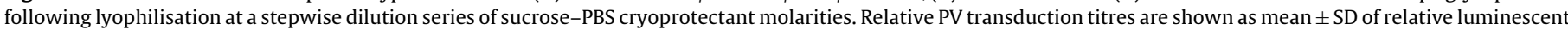
units per $\mathrm{ml}(\mathrm{RLU} / \mathrm{ml})$.

in serological assays. To address this issue, we reconstituted H5, RABV and MARV pseudotypes (immediately after lyophilisation, in the presence of $0.5 \mathrm{M}$ sucrose-PBS) with distilled, nuclease-free $\mathrm{H}_{2} \mathrm{O}$ and DMEM (with supplementation described in Section 2.1), before comparing their transduction ability into corresponding target cell lines with a titration assay (Fig. 6). Levels of pseudotype titre retention were very similar with either reconstitution solution, indicating that possible culture medium nutrient accumulation when using DMEM to reconstitute lyophilised pseudotypes does not have an adverse effect on pseudotype infectivity. However, water could viably be used as an alternative solution for resuspension of freeze-dried pseudotypes, but it is uncertain whether this would detrimentally affect the health of the target cell lines in titration and neutralisation assays, due to insufficient volumes of fresh DMEM.

The ability for lyophilised PVs to transduce target cells indicates that the influenza A, RABV and MARV envelope glycoproteins do not structurally deteriorate during the freeze-drying process, especially in the receptor-binding domains. However, in order to assess the structural integrity in the antigenic epitopes of the glycoproteins, neutralisation assays were also carried out using serum samples confirmed as antibody-positive against $\mathrm{H} 5$ and RABV strains. VNAb $\mathrm{IC}_{50}$ and $\mathrm{IC}_{90}$ titres (the reciprocal of the highest serum dilution still able to confer $50 \%$ and $90 \%$ virus neutralisation) were compared between lyophilised and immediately reconstituted H5 and RABV pseudotypes, and their 'fresh', unlyophilised counterparts, with no discernible reduction in the capability of antibody-mediated neutralisation observed (Table 1).

\section{Discussion}

It has been well-documented that retroviral pseudotypes are valid, reliable alternatives to wild-type virus for serological applications (Temperton and Wright, 2009). Advantageous qualities of the PVNA platform include the ability to conduct the assay in BSL-1

Table 1

Comparison of neutralising antibody titres against untreated and lyophilised pseudotyped lentiviruses. Half maximal inhibitory concentration $\left(\mathrm{IC}_{50}\right)$ and $90 \%$ inhibitory concentration ( $\mathrm{IC}_{90}$ ) values of confirmed antibody-positive antisera against H5 influenza and RABV pseudotypes before and after lyophilisation. VNAb titres were calculated using GraphPad Prism 5 software and are displayed as serum assay dilutions. Exact $\mathrm{IC}_{50}$ and $\mathrm{IC}_{90}$ values are also shown in parentheses.

\begin{tabular}{lcl}
\hline Pseudotype virus (PV) & IC $_{50}$ & IC $_{90}$ \\
\hline $\begin{array}{l}\text { H5 A/Vietnam/1194/2004 } \\
\quad \text { lyophilised }\end{array}$ & $2560-5120(4679)$ & $1280-2560(1390)$ \\
$\begin{array}{l}\text { H5 A/Vietnam/1194/2004 } \\
\quad \text { unlyophilised }\end{array}$ & $2560-5120(3919)$ & $1280-2560(1345)$ \\
RABV ERA - lyophilised & $40,960-81,920(44,072)$ & $5120-10,240(6381)$ \\
RABV ERA - unlyophilised & $20,480-40,960(27,324)$ & $5120-10,240(6222)$ \\
\hline
\end{tabular}




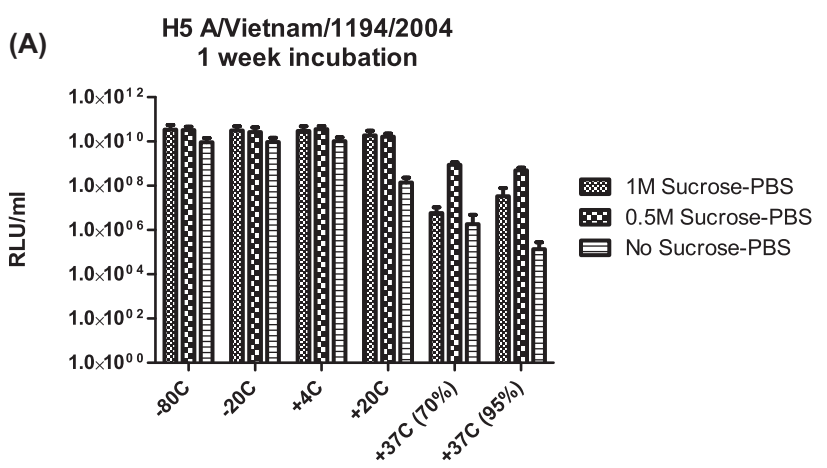

Temperature $\left({ }^{\circ} \mathrm{C}\right)[\&$ Relative Humidity $(\%)]$

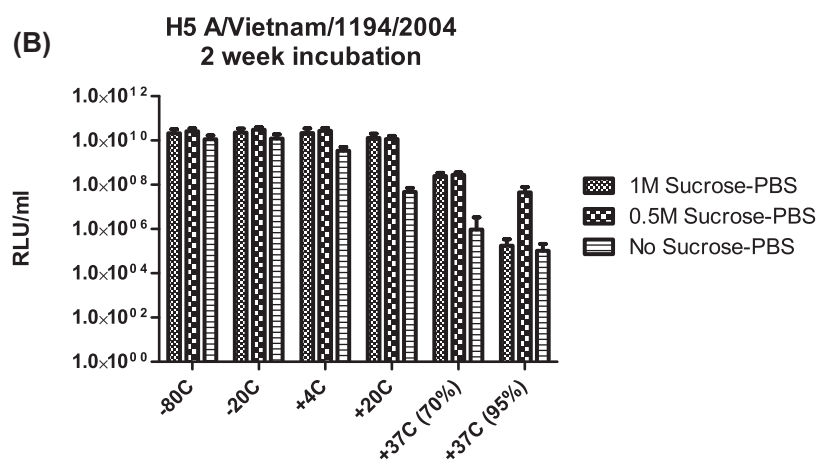

Temperature $\left({ }^{\circ} \mathrm{C}\right)[\&$ Relative Humidity $(\%)]$

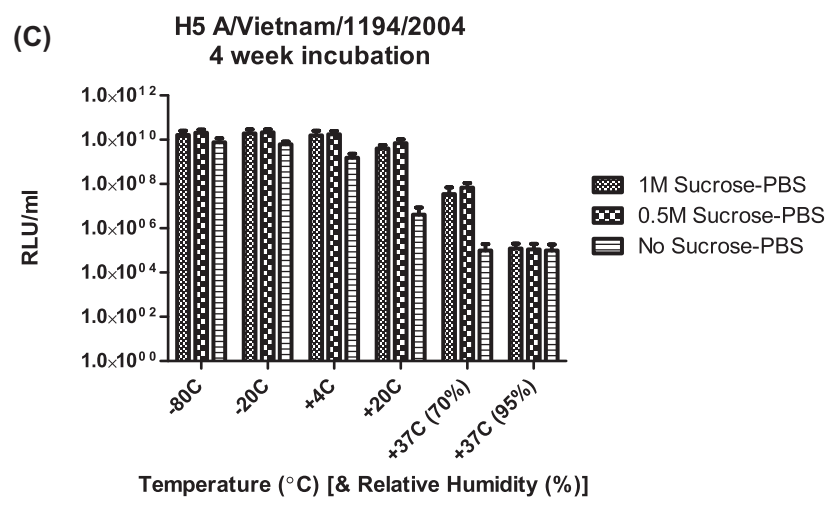

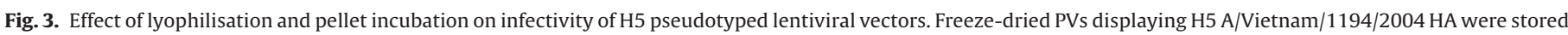

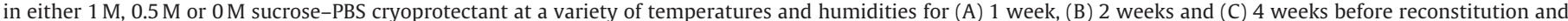
employment in a titration assay. Pseudotype transduction titres are displayed as mean \pm SD of relative luminescent units per $\mathrm{ml}$ ( $\mathrm{RLU} / \mathrm{ml}$ ).

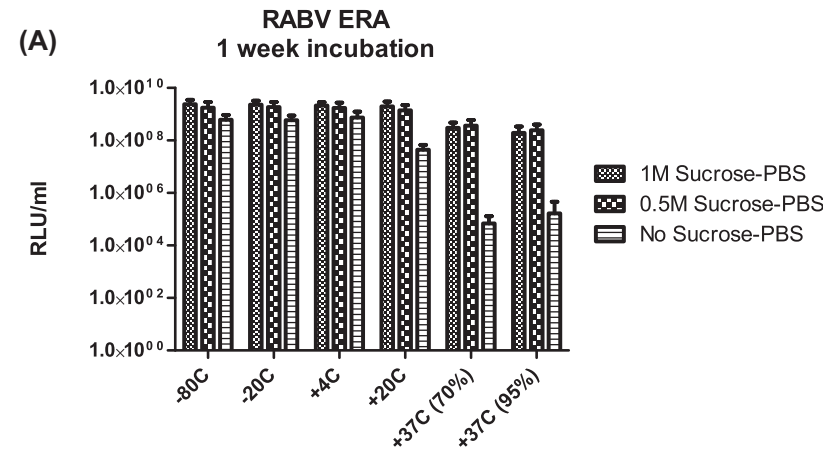

Temperature $\left({ }^{\circ} \mathrm{C}\right)[\&$ Relative Humidity $(\%)]$

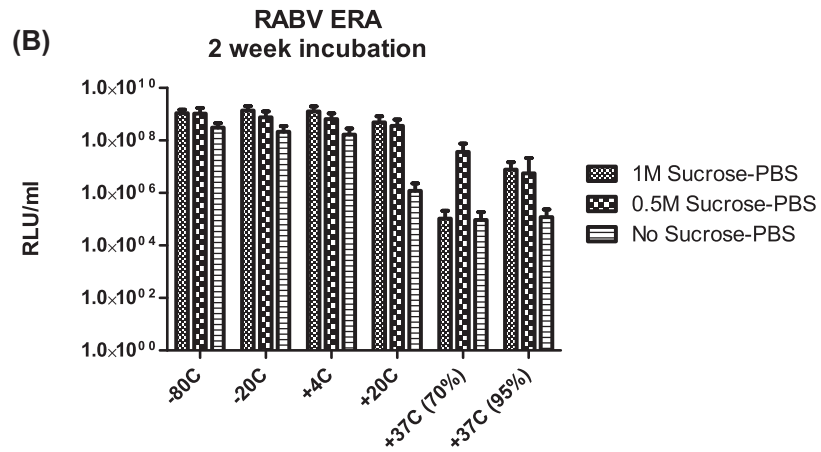

Temperature $\left({ }^{\circ} \mathrm{C}\right)[\&$ Relative Humidity $(\%)]$

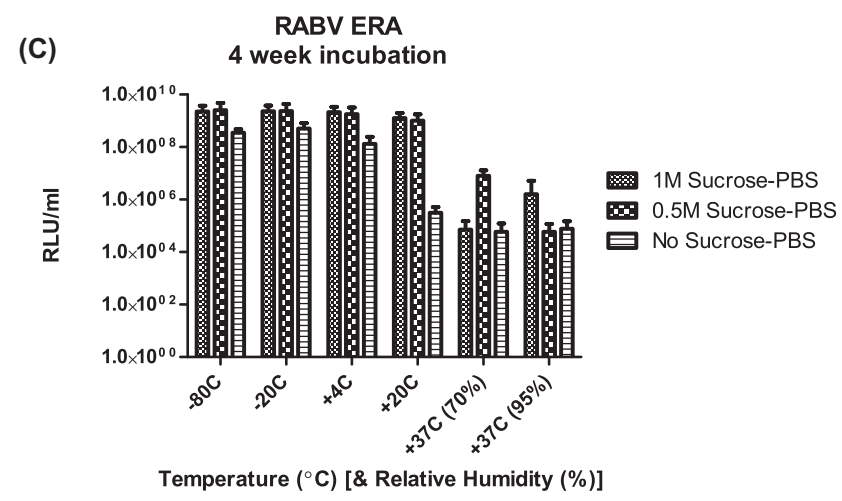

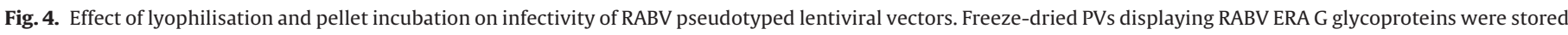

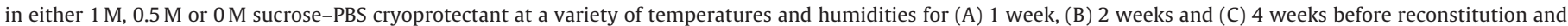
employment in a titration assay. Pseudotype transduction titres are expressed as mean $\pm \mathrm{SD}$ of relative luminescent units per $\mathrm{ml}(\mathrm{RLU} / \mathrm{ml}$ ). 


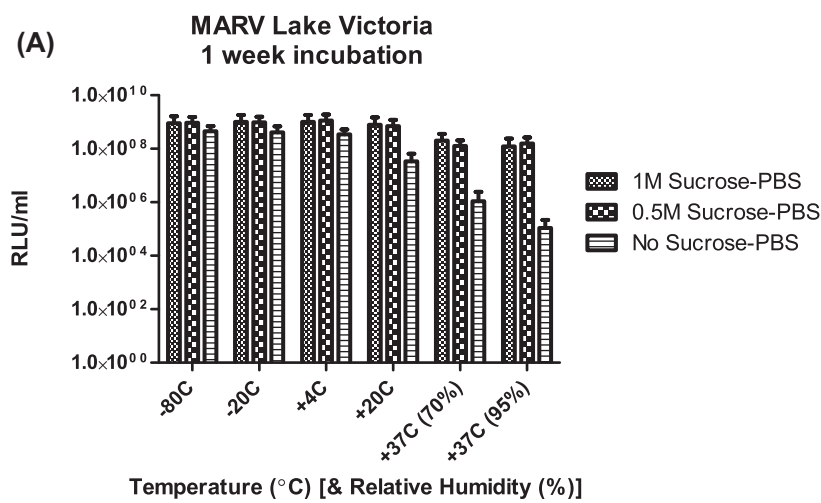

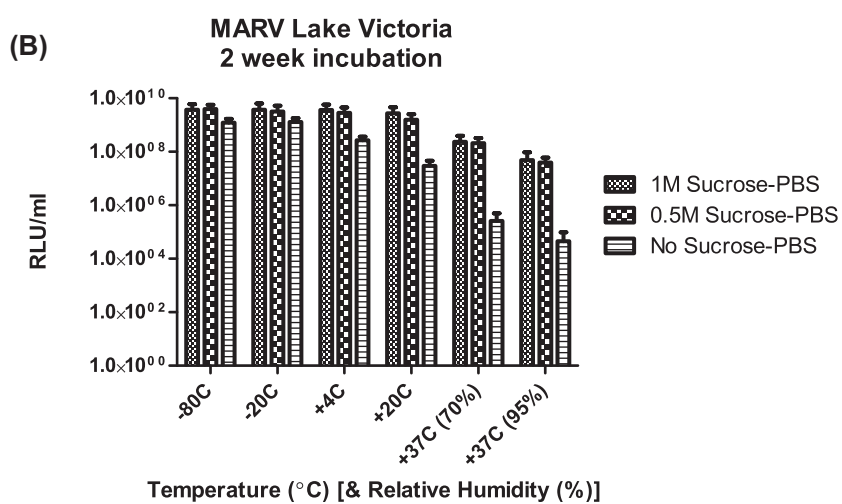

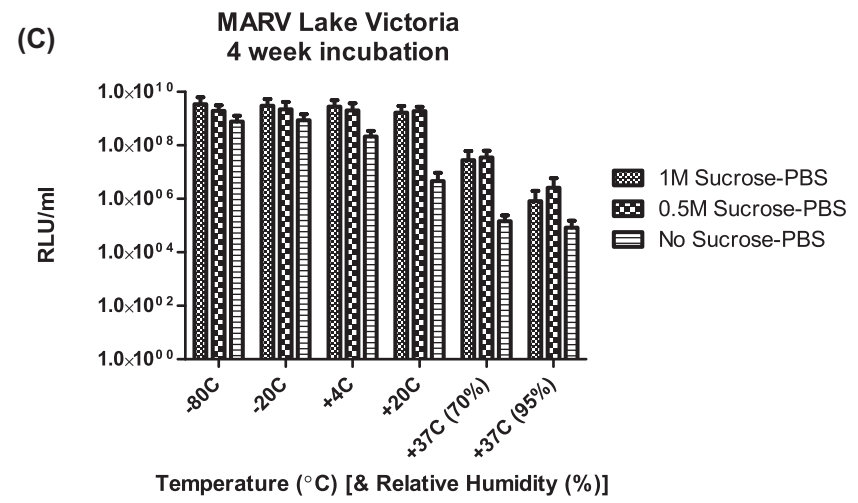

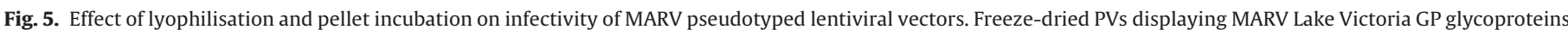

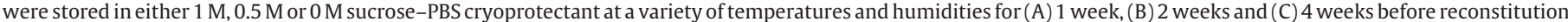
and employment in a titration assay. Pseudotype transduction titres are shown as mean \pm SD of relative luminescent units per $\mathrm{ml}$ (RLU/ml).

laboratories, as well as the availability to perform multiplex assays and incorporate 'low-cost' reporter genes into pseudoparticles, which all reduce the cost implications and increase the potential ubiquity of pseudotyping (Wright et al., 2009, 2010; Kaku et al., 2012). However, the current necessity to store aliquots of PV supernatant at $-80^{\circ} \mathrm{C}$ and to maintain the cold-chain during PV transportation present serious monetary obstacles for laboratories to acquire such reagents, especially if on a limited budget.

The viability of lyophilisation was demonstrated as an alternative, cost-effective state for the storage and distribution of pseudotype viruses. In the presence of cryoprotectant, H5 influenza, rabies and Marburg PV supernatant retain very high levels of infectivity following freeze-drying and reconstitution. Subsequent freeze-dried pseudotype pellets can generally withstand incubation for 4 weeks at a range of temperatures up to $37^{\circ} \mathrm{C}$, and incubation in a 'tropical climate' $\left(37^{\circ} \mathrm{C}\right.$ and $95 \%$ relative humidity) for 2 weeks, whilst maintaining a viral titre sufficient for employment in downstream neutralisation assays. This confirms the stability and glycoprotein integrity of lyophilised PVs throughout environmental conditions likely to be experienced within the production, dissemination and storage of a PVNA-based kit. Furthermore, both $\mathrm{H} 5$ influenza and rabies reconstituted pseudotypes were neutralised by VNAb-positive serum samples to similar potencies as their 'fresh', unlyophilised counterparts, indicating that antigenic epitopes on each virus glycoprotein do not structurally deteriorate during lyophilisation, thus ratifying the suitability of freeze-dried PVs from a serological viewpoint.

With regard to the wider implications for PVNA-based serology kits, the findings reported in this study are also encouraging. The survival and usability of somatic cells after freeze-drying and reconstitution has already been established, with positive implications for many areas of biomedicine (Loi et al., 2008). Indeed, the opportunity to produce samples of pseudotype virus and candidate cell line as stable, dried pellets would considerably facilitate

\section{H5 AVietnam/1194/2004, RABV ERA and MARV Lake Victoria $\mathrm{H}_{2} \mathrm{O}$ and DMEM reconstitution}

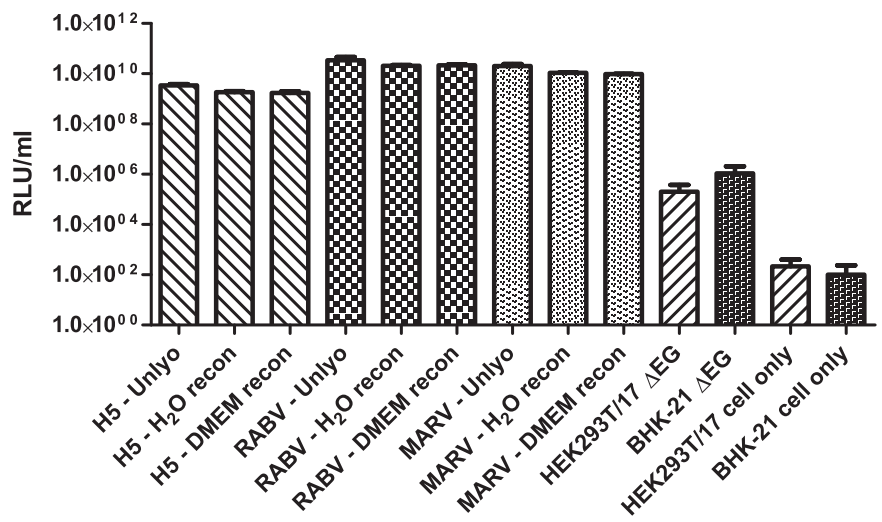

Fig. 6. Influence of reconstitution solution on H5, RABV and MARV pseudotyped lentiviral vectors. Freeze-dried PVs with influenza H5 A/Vietnam/1194/2004 HA RABV ERA G or MARV Lake Victoria GP envelope glycoproteins were reconstituted in either distilled, nuclease-free $\mathrm{H}_{2} \mathrm{O}$ or supplemented DMEM culture medium before utilisation in a titration assay. Pseudotype transduction titres are shown as mean \pm SD of relative luminescent units per $\mathrm{ml}(\mathrm{RLU} / \mathrm{ml})$. Unlyophilised pseudotype positive controls, and $\Delta$ envelope glycoprotein, HEK293T/17 cell only and BHK-21 cell only negative controls are also shown. 
global distribution of a multi-component PVNA kit, at a fraction of the current expenditure for overseas shipping and storage on dry ice. Additionally, the high cost of purchasing frozen cell line ampoules from certified repositories can be avoided by incorporating lyophilised cells into such a kit. Overall, the utilisation of PVNA-based kits would significantly ameliorate logistic dilemmas surrounding vaccine evaluation and serological surveillance, especially for laboratories situated in resource-poor countries where many emerging viral infections are prevalent.

Virus lyophilisation as a stable means of storage is certainly not a novel phenomenon, with the process being acknowledged for decades (Tyrrell and Ridgwell, 1965). Studies involving wild-type virus freeze-drying generally concur with this one in several aspects by, for instance, demonstrating the ability to store lyophilised foot and mouth disease virus at $4{ }^{\circ} \mathrm{C}$ for 1 year (Fellowes, 1965) and freeze-dried poliovirus preparations at $37^{\circ} \mathrm{C}$ for 5 days (Berge et al., 1971). Infectivity tests were also undertaken on pseudorabies virus lyophilised in a number of suspension media, with glutamate formulations mixed with sucrose or dextran proving the most cryoprotective (Scott and Woodside, 1976). Furthermore, the viability of freeze-dried viral vector formulations has been investigated for gene therapy applications. Retroviral vectors have recovered with more than $90 \%$ infectivity post-lyophilisation in the presence of sucrose cryoprotectant (Shin et al., 2010), with adenoviral vectors only showing negligible drops in titre following freeze-drying and storage at ambient temperatures (Croyle et al., 2001). Likewise, lyophilsed influenza virosomes retained both structure and function after 12 weeks' storage at $4{ }^{\circ} \mathrm{C}$ (Wilschut et al., 2007). In comparison, pseudotyped retroviral vectors rapidly decreased in titre following three to five freeze-thaw cycles (Higashikawa and Chang, 2001), which further reinforces how preferable lyophilisation is for employment in VNAb-based serological kits.

Relative humidity (RH) plays a pivotal role in storage stability of dried pseudotype pellets, with high RH levels proving detrimental to PV recovery and transduction potential. Certainly for wild-type influenza virus, it has been confirmed that both virus transmission and infectivity are significantly decreased in highly humid atmospheres, which could be an attributive factor for its seasonal fluctuation (Lowen et al., 2007; Noti et al., 2013). It has previously been postulated that viruses with a high lipid content are more sensitive to high RH (Assar and Block, 2001).

Investigation of further parameters would be necessary before a robust, reliable PVNA-based kit could be trialled and clinically utilised. Firstly, existing data would need to be extrapolated by testing freeze-dried pellet storage stability over longer durations i.e. six months, one year and three years, as well as comparing other candidate suspending media to sucrose-PBS to ensure maximum efficiency of cryoprotection. Employing freeze-dried pseudotypes in PVNAs against larger panels of sera, before drawing comparisons against not only unlyophilised pseudotypes, but also established serological assays using live virus, would be vital to assess accordance in VNAb titres between assays. To increase PVNA kit flexibility and customisation, it would also be important to assess the sensitivity of other commonly used pseudotype virus cores to lyophilisation and subsequent stability studies. Another consideration is to accurately simulate conditions during an international transit journey, thus ascertaining the ability for lyophilised PVs to cope with harsh temperature and atmospheric fluctuations between, for example, an aeroplane cargo deck and tropical climate conditions.

\section{Conclusion}

In this study, it is shown $\mathrm{H} 5$ influenza, rabies and Marburg pseudotype viruses can be stably stored in a lyophilised state for 4 weeks at temperatures up to $37^{\circ} \mathrm{C}$, in the presence of at least $0.5 \mathrm{M}$ sucrose-PBS as a cryoprotectant, and retain much of their infectivity once reconstituted and employed in virus neutralisation assays. This confirms the viability of producing a freeze-dried PVNA-based kit, which would considerably facilitate the execution of vaccine evaluation and sero-surveillance studies, especially in countries without access to BSL-3/-4 containment laboratories or constant cold-chain storage facilities, and ultimately permit the development of improved serological control measures for many emerging viral infections.

\section{Acknowledgements}

The authors wish to thank Janet Daly, Barnabas King and Francesca Ferrara for constructive discussion throughout this study.

\section{References}

Assar, S.K., Block, S.S, 2001. Survival of microorganisms in the environment. In: Block, S.S. (Ed.), Disinfection, Sterilization and Preservation. Lippincott Williams and Wilkins, Phildelphia, Pennsylvania (PA), USA, pp. 1221-1242.

Berge, T.O., Jewett, R.L., Blair, W.O., 1971. Preservation of enteroviruses by freezedrying. Appl. Microbiol. 22, 850-853.

Brauburger, K., Hume, A.J., Mühlberger, E., Olejnik, J., 2012. Forty-five years of Marburg virus research. Viruses 4, 1878-1927.

Capecchi, B., Fasolo, A., Alberini, I., Baudner, B., Crotta, S., Temperton, N.J., Montomoli, E., Del Guidice, G., Rappuoli, R., 2008. Use of pseudotyped particles expressing influenza A/Vietnam/1194/2004 hemagglutinin in neutralization assays. In: Katz, J. (Ed.), Options for the Control of Influenza VI. International Medical Press, London, pp. 303-305.

Cliquet, F., Aubert, M., Sagné, L., 1998. Development of a fluorescent antibody virus neutralisation test (FAVN test) for the quantitation of rabies-neutralising antibody. J. Immunol. Methods 212, 79-87.

Cox, R.J., Mykkeltvedt, E., Robertson, J., Haaheim, L.R., 2002. Non-lethal viral challenge of influenza haemagglutinin and nucleoprotein DNA vaccinated mice results in reduced viral replication. Scand. J. Immunol. 55, 14-23.

Croyle, M.A., Cheng, X., Wilson, J.M., 2001. Development of formulations that enhance physical stability of viral vectors for gene therapy. Gene Ther. 8, 1281-1290.

Demaison, C., Parsley, K., Brouns, G., Scherr, M., Battmer, K., Kinnon, C., Grez, M., Thrasher, A.J., 2002. High-level transduction and gene expression in hematopoietic repopulating cells using a human immunodeficiency [correction of imunodeficiency] virus type 1-based lentiviral vector containing an internal spleen focus forming virus promoter. Hum. Gene Ther. 13, 803-813.

Desvaux, S., Garcia, J.M., Nguyen, T.D., Reid, S.A., Bui, N.A., Roger, F., Fenwick, S., Peiris, J.S.M., Ellis, T., 2012. Evaluation of serological tests for H5N1 avian influenza on field samples from domestic poultry populations in Vietnam: consequences for surveillance. Vet. Microbiol. 156, 277-284.

Fellowes, O.N., 1965. Freeze-drying of foot-and-mouth disease virus and storage stability of the infectivity of dried virus at $4{ }^{\circ} \mathrm{C}$. Appl. Microbiol. 13, 496-499.

Gao, R., Cao, B., Hu, Y., Feng, Z., Wang, D., Hu, W., Chen, J., Jie, Z., Qiu, H., Xu, K., Xu, X., Lu, H., Zhu, W., Gao, Z., Xiang, N., Shen, Y., He, Z., Gu, Y., Zhang, Z., Yang, Y., Zhao, X., Zhou, L., Li, X., Zou, S., Zhang, Y., Li, X., Yang, L., Guo, J., Dong, J., Li, Q., Dong, L., Zhu, Y., Bai, T., Wang, S., Hao, P., Yang, W., Zhang, Y., Han, J., Yu, H., Li, D., Gao, G.F., Wu, G., Wang, Y., Yuan, Z., Shu, Y., 2013. Human infection with a novel avian-origin influenza A (H7N9) virus. N. Engl. J. Med. 368, 1888-1897.

Higashikawa, F., Chang, L., 2001. Kinetic analyses of stability of simple and complex retroviral vectors. Virology 280, 124-131.

Jackson, A.C., 2013. Current and future approaches to the therapy of human rabies. Antiviral Res. 99, 61-67.

Kaku, Y., Noguchi, A., Marsh, G.A., Barr, J.A., Okutani, A., Hotta, K., Bazartseren, B., Fukushi, S., Broder, C.C., Yamada, A., Inoue, S., Wang, L.-F., 2012. Second generation of pseudotype-based serum neutralization assay for Nipah virus antibodies: sensitive and high-throughput analysis utilizing secreted alkaline phosphatase. J. Virol. Methods 179, 226-232.

Kishishita, N., Takeda, N., Anuegoonpipat, A., Anantapreecha, S., 2013. Development of a pseudotyped-lentiviral-vector-based neutralization assay for chikungunya virus infection. J. Clin. Microbiol. 51, 1389-1395.

Loi, P., Matsukawa, K., Ptak, G., Clinton, M., Fulka, J., Nathan, Y., Arav, A., 2008. Freezedried somatic cells direct embryonic development after nuclear transfer. PLOS ONE 3, e2978

Lowen, A.C., Mubareka, S., Steel, J., Palese, P., 2007. Influenza virus transmission is dependent on relative humidity and temperature. PLOS Pathog. 3, 1470-1476.

Mather, S., Scott, S., Temperton, N., Wright, E., King, B., Daly, J., 2013. Current progress with serological assays for exotic emerging/re-emerging viruses. Future Virol. 8, 745-755. 
Molesti, E., Milani, A., Terregino, C., Cattoli, G., Temperton, N.J., 2013. Comparative serological assays for the study of h5 and h7 avian influenza viruses. Influenza Res. Treat. 2013, 286158, http://dx.doi.org/10.1155/2013/286158.

Noti, J.D., Blachere, F.M., McMillen, C.M., Lindsley, W.G., Kashon, M.L., Slaughter, D.R., Beezhold, D.H., 2013. High humidity leads to loss of infectious influenza virus from simulated coughs. PLOS ONE 8, e57485.

Papenburg, J., Baz, M., Hamelin, M.-È., Rhéaume, C., Carbonneau, J., Ouakki, M. Rouleau, I., De Serres, G., Boivin, G., 2011. Evaluation of serological diagnostic methods for the 2009 pandemic influenza A (H1N1) virus. Clin. Vaccine Immunol. 18, 520-522.

Pear, W.S., Nolan, G.P., Scott, M.L., Baltimore, D., 1993. Production of high-titer helper-free retroviruses by transient transfection. Proc. Natl. Acad. Sci. U. S. A. 90, 8392-8396.

Scott, E.M., Woodside, W., 1976. Stability of pseudorabies virus during freeze-drying and media. In: Stability of Pseudorabies Virus During Freeze-Drying and Storage: Effect of Suspending Media.

Scott, S., Molesti, E., Temperton, N., Ferrara, F., Böttcher-Friebertshäuser, E., Daly, J., 2012. The use of equine influenza pseudotypes for serological screening. J. Mol. Genet. Med. 6, 304-308.

Shin, S., Salvay, D., Shea, L., 2010. Lentivirus delivery by adsorption to tissue engineering scaffolds. J. Biomed. Mater. 93, 1252-1259.

Stoker, M., MacPherson, I., 1964. Syrian hamster fibroblast cell line BHK21 and its derivatives. Nature 203, 1355-1357.

Temperton, N.J., Hoschler, K., Major, D., Nicolson, C., Manvell, R., Hien, V.M., Ha, D.Q., de Jong, M., Zambon, M., Takeuchi, Y., Weiss, R.A., 2007. A sensitive retroviral pseudotype assay for influenza H5N1-neutralizing antibodies. Influenza Other Respir. Viruses 1, 105-112.

Temperton, N.J., Wright, E., 2009. Retroviral pseudotypes. In: Encyclopedia of Life Sciences. John Wiley \& Sons, Chichester.

Terregino, C., Toffan, A., Cilloni, F., Monne, I., Bertoli, E., Castellanos, L., Amarin, N., Mancin, M., Capua, I., 2010. Evaluation of the protection induced by avian influenza vaccines containing a 1994 Mexican H5N2 LPAI seed strain against a 2008 Egyptian H5N1 HPAI virus belonging to clade 2.2.1 by means of serological and in vivo tests. Avian Pathol. 39, 215-222.

To, K.K.W., Tsang, A.K.L., Chan, J.F.W., Cheng, V.C.C., Chen, H., Yuen, K.-Y., 2014. Emergence in China of human disease due to avian influenza A(H10N8) - cause for concern? J. Infect. 68, 205-215.
Tong, S., Zhu, X., Li, Y., Shi, M., Zhang, J., Bourgeois, M., Yang, H., Chen, X. Recuenco, S., Gomez, J., Chen, L.-M., Johnson, A., Tao, Y., Dreyfus, C., Yu, W., McBride, R., Carney, P.J., Gilbert, A.T., Chang, J., Guo, Z., Davis, C.T., Paulson, J.C., Stevens, J., Rupprecht, C.E., Holmes, E.C., Wilson, I.A., Donis, R.O., 2013. New world bats harbor diverse influenza A viruses. PLOS Pathog. 9, e1003657, http://dx.doi.org/10.1371/journal.ppat.1003657.

Tyrrell, D.A., Ridgwell, B., 1965. Freeze-drying of certain viruses. Nature 206, $115-116$.

Wei, S.-H., Yang, J.-R., Wu, H.-S., Chang, M.-C., Lin, J.-S., Lin, C.-Y., Liu, Y.-L., Lo, Y.C., Yang, C.-H., Chuang, J.-H., Lin, M.-C., Chung, W.-C., Liao, C.-H., Lee, M.-S. Huang, W.-T., Chen, P.-J., Liu, M.-T., Chang, F.-Y., 2013. Human infection with avian influenza A H6N1 virus: an epidemiological analysis. Lancet Respir. Med. $1,771-778$.

WHO, 2010. Pandemic (H1N1) 2009 Update 112. Global Alert and Response, Geneva. WHO, 2013. Rabies Fact Sheet No. 99, Geneva.

Wilschut, J., de Jonge, J., Huckriede, A., Amorij, J.-P., Hinrichs, W.L.J., Frijlink, H.W. 2007. Preservation of influenza virosome structure and function during freezedrying and storage. J. Liposome Res. 17, 173-182.

Wright, E., Hayman, D.T.S., Vaughan, A., Temperton, N.J., Wood, J.L.N., Cunningham, A.A., Suu-Ire, R., Weiss, R.A., Fooks, A.R., 2010. Virus neutralising activity of African fruit bat (Eidolon helvum) sera against emerging lyssaviruses. Virology 408, 183-189.

Wright, E., McNabb, S., Goddard, T., Horton, D.L., Lembo, T., Nel, L.H., Weiss, R.A., Cleaveland, S., Fooks, A.R., 2009. A robust lentiviral pseudotype neutralisation assay for in-field serosurveillance of rabies and lyssaviruses in Africa. Vaccine 27, 7178-7186

Wright, E., Temperton, N.J., Marston, D.A., McElhinney, L.M., Fooks, A.R., Weiss, R.A., 2008. Investigating antibody neutralization of lyssaviruses using lentiviral pseudotypes: a cross-species comparison. J. Gen. Virol. 89, 2204-2213.

Yuen, K.Y., Chan, P.K., Peiris, M., Tsang, D.N., Que, T.L., Shortridge, K.F., Cheung, P.T. To, W.K., Ho, E.T., Sung, R., Cheng, A.F., 1998. Clinical features and rapid vira diagnosis of human disease associated with avian influenza A H5N1 virus. Lancet $351,467-471$.

Zufferey, R., Nagy, D., Mandel, R.J., Naldini, L., Trono, D., 1997. Multiply attenuated lentiviral vector achieves efficient gene delivery in vivo. Nat. Biotechnol. 15, 871-875. 